\title{
RESISTENCIA TRANSMITIDA EN VIH-1 DE PACIENTES PROVENIENTES DE NUEVE DEPARTAMENTOS DEL PERÚ
}

\author{
Carlos A. Yabar(1,2,a,b, Giovanny F. Vilcarino(1),a, Susan Espetia(1)1,a,c,

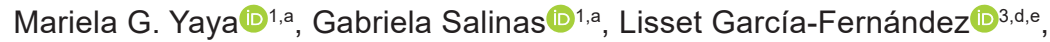 \\ Rubén D. Vásquez Becerra ${ }^{4, d, e}$, Carlos Benites ${ }^{5, d}$, Daniel Santos-Anaya (1) 1,f, \\ Edgardo Mamani(1,a,g, Maribel Acuña@1,a, Soledad Romero@1,a, Fany Cárdenas (11,a \\ ${ }^{1}$ Laboratorio de VTS/VIH-SIDA, Instituto Nacional de Salud, Lima, Perú. \\ 2 Universidad San Martín de Porres, Lima, Perú. \\ ${ }^{3}$ Ministerio de Salud del Perú, Lima, Perú. \\ ${ }^{4}$ Hospital Nacional María Auxiliadora, Lima, Perú. \\ ${ }^{5}$ Dirección de Prevención y Control de VIH-SIDA, Enfermedades de Transmisión Sexual y Hepatitis, Ministerio de Salud, \\ Lima, Perú. \\ a Biólogo/a; ${ }^{b}$ doctor en Ciencias Biológicas; ${ }^{\mathrm{c}}$ magíster en Microbiología; ${ }^{\mathrm{d}}$ médica/o cirujana/o; ${ }^{\mathrm{e}}$ magíster en Salud Pública; \\ f tecnólogo médico; ${ }^{g}$ magíster en Biotecnología.
}

\section{RESUMEN}

El estudio tuvo como objetivo determinar la resistencia transmitida (RT) del virus de la inmunodeficiencia humana (VIH) en pacientes procedentes de nueve departamentos del Perú. Para ello, se realizó un estudio descriptivo en 132 adultos que aceptaron participar mediante un consentimiento informado. Se colectaron muestras de sangre para realizar el recuento de células CD4/CD8, determinar la carga viral y la genotipificación del VIH. Se recabó información socioepidemiológica de los participantes mediante encuestas. Los resultados revelaron una frecuencia de RT de 9,8\% (13/132). Los resultados del estudio ayudarán a mejorar los programas de intervención y monitoreo de la resistencia a los antirretrovirales en el país.

Palabras clave: Genotipo; Farmacorresistencia; VIH (Fuente: DeCS BIREME).

\section{TRANSMITTED RESISTANCE IN HIV-1 OF PATIENTS FROM NINE DEPARTMENTS OF PERU}

\begin{abstract}
The main aim of this research is to identify the transmitted resistance (RT) of the Human Immunodeficiency Virus (HIV) in subjects from nine cities of Peru. For this, a descriptive and cross-sectional study was carried out in 135 adult subjects who agreed to participate through an informed consent. Blood samples were collected to perform the CD4 / CD8 cell count, viral load and HIV Genotyping. Socio-epidemiological information was collected from the participants through surveys. The results revealed a RT frequency of $9.8 \%$ (13/132). The information from this research might help improve the intervention and monitoring programs for antiretroviral resistance in the country.
\end{abstract}

Citar como: Yabar CA, Vilcarino GF, Espetia S, Yaya MG, Salinas G, García-Fernández L, et al. Resistencia transmitida en VIH-1 de pacientes provenientes de nueve departamentos del Perú. Rev Peru Med Exp Salud Publica. 2021;38(1):7782. doi: https://doi.org/10.17843/ rpmesp.2021.381.5527.

Correspondencia: Carlos Augusto Yabar; Av. Defensores del Morro 2268, Chorrillos, Lima, Perú; cyabar@ins.gob.pe

Recibido: 03/04/2020 Aprobado: 07/10/2020 En línea: 20/12/2020
Keywords: Genotype; Drug resistance; HIV (Source: MeSH NLM).

\section{INTRODUCCIÓN}

El VIH/sida afecta a más 36 millones de personas en todo el mundo; a pesar de que la pandemia sigue en aumento, la tasa de mortalidad por sida ha disminuido hasta un $34 \%$, debido principalmente al aumento de la cobertura de la terapia antirretroviral (TAR) ${ }^{(1)}$.

Cabe indicar que cuando se transmite un genotipo resistente de VIH a una persona sana o previamente infectada, dará lugar a la resistencia transmitida $(\mathrm{RT})^{(2)}$, la cual se relaciona con riesgo de falla virológica en pacientes con inicio de TAR ${ }^{(3)}$. En ese sentido, es importante identificar y realizar la vigilancia de la RT a través de pruebas de genotipificación de VIH ${ }^{(2)}$.

Respecto a la prevalencia de la RT en países de Latinoamérica y el Caribe (LAC), una reciente revisión sistemática realizada a partir de 81 estudios entre 2006 y 2015 reveló una frecuencia 
de $7,7 \%{ }^{(4)}$. Asimismo, una revisión realizada a partir de 358 estudios en 64 países reveló que la prevalencia de la RT en LAC fue de $12 \%$, superior a Asia (2\%) y África (9\%) ${ }^{(5)}$.

Dentro de los factores socioepidemiológicos relacionados con la RT, se ha demostrado asociación con población de hombres que tienen sexo con otros hombres (HSH) ${ }^{(6)}$, con falla virológica ${ }^{(7)}$ y con infecciones de transmisión sexual (ITS) ${ }^{(8)}$, lo cual sugiere que no existe necesariamente un factor común asociado a la RT.

La mayoría de los estudios de RT en el Perú se realizaron en la ciudad de Lima y revelaron prevalencias del $1 \%$ al $14 \%{ }^{(10-12)}$. Estas diferencias en la prevalencia de la RT podrían estar relacionadas con el período del estudio, diseño metodológico, tipo de prueba genotípica utilizada, tamaño, origen y características de los participantes en cada estudio.

Asimismo, se desconoce si la frecuencia de RT en Lima es similar a la de otros departamentos del Perú, ya que la dinámica de transmisión en la capital es diferente en comparación a la del resto del país ${ }^{(13)}$. Además, no se han descrito los factores socioepidemiológicos en pacientes peruanos con RT. Por tanto, el objetivo de este estudio fue estimar la frecuencia de la RT en pacientes con VIH/sida sin TAR de nueve departamentos del Perú. Adicionalmente se describieron los factores socioepidemiológicos presentes en los participantes.

\section{EL ESTUDIO}

Se realizó un estudio de tipo descriptivo y transversal, el cual se ejecutó entre julio de 2014 y diciembre de 2015, en 29 de los 79 establecimientos públicos de salud del Ministerio de Salud del Perú. Se seleccionaron estos centros por cubrir la atención de un $87,9 \%$ de pacientes con inicio de TAR a nivel nacional ${ }^{(13)}$ y corresponden a nueve departamentos del Perú (Arequipa, Ica, Junín, Lambayeque, La Libertad, Lima, Loreto, Piura, y San Martín).

El tamaño de la muestra se calculó a través del programa estadístico Epidat versión 3.1 considerando una prevalencia de RT de $7,7 \%{ }^{(4)}$, asumiendo un error absoluto del 5\%, un 95\% de nivel de confianza y una tasa de pérdida de datos de $20 \%$, con lo cual se estableció incluir, al menos, 132 pacientes. En la Tabla 1 se detalla la distribución de participantes por departamentos. El tipo de muestreo aplicado fue no probabilístico consecutivo, incluyó pacientes de ambos sexos con al menos 18 años, diagnóstico confirmado de VIH por ELISA, Western Blot, ensayo inmunoblot lineal (LIA) o inmunofluorescencia indirecta (IFI), sin TAR previo. No se consideraron a personas con problemas mentales ni mujeres en gestación.

Para el reclutamiento de los pacientes, se tomaron en cuenta los principios éticos establecidos durante el procedimiento de consentimiento informado (CI). Asimismo, los participantes completaron una encuesta escrita para recabar

\section{MENSAJES CLAVE}

Motivación para realizar el estudio: El estudio surge a raíz de la escasa información a nivel nacional de la situación de la resistencia trasmitida (RT) a los antirretrovirales en la población peruana.

Principales hallazgos: En este estudio se demostró que la RT es moderada según clasificación de la Organización Mundial de la Salud (OMS).

Implicancias: El mejoramiento de las políticas públicas de tratamiento y campañas de educación sexual, con el fin de ayudar a disminuir los casos de RT en todo el Perú.

información socioepidemiológica y clínica de estos. Seguidamente se tomaron muestras de sangre periférica que se trasladaron al Laboratorio de Referencia Nacional de VTS/ VIH-SIDA del Instituto Nacional de Salud (INS) de Lima en cajas selladas y rotuladas indicando el lugar de procedencia. La información de los pacientes se digitalizó en una base de datos en Excel con acceso restringido y a cargo del investigador principal mientras que los CI fueron archivados bajo llave para asegurar la confidencialidad de los participantes.

Para la genotipificación de VIH se amplificó una región de 1020 pb mediante dos rondas de PCR ${ }^{(14)}$ y se determinó la secuencia por el método de Sanger usando el analizador genético ABI 3500 XL (Thermo Fisher Scientific ${ }^{\oplus}$, Reino Unido). Las muestras que no amplificaron por la plataforma In House, fueron manejadas usando el método comercial Trugene ${ }^{\circledast}$ (Siemens Healthcare Diagnostics Inc. Deerfield, Illinois, EUA). La secuencia consenso se obtuvo mediante el programa RECall (beta v3.05) ${ }^{(15)}$.

Para realizar el análisis de las mutaciones de resistencia, las secuencias se ingresaron al programa HIVdb Program (https://hivdb.stanford.edu/hivdb/by-mutations/) de la base de datos del HIV Drug Resistance Database de la Universidad de Stanford versión 8.8, mientras que el control de calidad de las secuencias se realizó con la Herramienta de Control de Calidad de la Organización Mundial de la Salud (OMS) (https://pssm.cfenet.ubc.ca/who_qc).

La carga viral se determinó mediante un sistema automatizado (Cobas) de PCR en tiempo real usando sondas Taqman (Roche, Branchburg, EUA), mientras que el recuento de CD4/CD8 se realizó mediante citometría de flujo por intermedio del kit BD Multitest ${ }^{\mathrm{TN}}$ IMK y el citómetro Facscanto II (BD).

Los datos recolectados se procesaron con el paquete estadístico Stata v14,2 (Stata Corporation, College Station, Texas, EUA), con el cual se obtuvieron resultados descriptivos mediante proporciones para las variables cualitativas (departamento de residencia, estado clínico, infección de transmisión sexual, tipo de ITS y mutación identificada) y de media con desviación estándar para las de naturaleza cuanti- 
Tabla 1. Características socioepidemiológicas y virológicas de la muestra de estudio.

\begin{tabular}{|c|c|}
\hline Características & n (\%) / media \pm DE \\
\hline Edad & $35,1 \pm 10,77$ \\
\hline \multicolumn{2}{|l|}{ Sexo } \\
\hline Femenino & $26(19,3)$ \\
\hline Masculino & $109(80,7)$ \\
\hline \multicolumn{2}{|l|}{ Departamento de residencia } \\
\hline Arequipa & $6(4,4)$ \\
\hline Ica & $3(2,2)$ \\
\hline Junín & $10(7,4)$ \\
\hline La Libertad & $7(5,2)$ \\
\hline Lambayeque & $16(11,9)$ \\
\hline Lima/Callao & $41(30,4)$ \\
\hline Loreto & $17(12,6)$ \\
\hline Piura & $3(2,2)$ \\
\hline San Martín & $32(23,7)$ \\
\hline \multicolumn{2}{|l|}{ Años de diagnóstico de $\mathrm{VIH}^{\dagger}$} \\
\hline Arequipa & $2,00 \pm 1,79$ \\
\hline Ica & $4,00 \pm 5,66$ \\
\hline Junín & $1,40 \pm 1,26$ \\
\hline La Libertad & $1,86 \pm 1,86$ \\
\hline Lambayeque & $2,56 \pm 1,41$ \\
\hline Lima/Callao & $1,90 \pm 2,35$ \\
\hline Loreto & $1,25 \pm 2,14$ \\
\hline Piura & $1,67 \pm 1,15$ \\
\hline San Martín & $0,90 \pm 0,49$ \\
\hline Total & $1,67 \pm 1,89$ \\
\hline \multicolumn{2}{|l|}{ Estado clínico } \\
\hline Sida & $45(33,3)$ \\
\hline No sida & $78(57,8)$ \\
\hline No establecido & $12(8,9)$ \\
\hline \multicolumn{2}{|l|}{ Infección de transmisión sexual } \\
\hline Sí & $43(33,1)$ \\
\hline No & $81(62,3)$ \\
\hline No precisa & $6(4,6)$ \\
\hline \multicolumn{2}{|l|}{ Estado virológico e inmunológico } \\
\hline Carga viral (copias/mL) & $247150,8 \pm 39194,7$ \\
\hline Recuento de células CD4 (células/ $\mu \mathrm{L}$ ) & $282,6 \pm 222,7$ \\
\hline
\end{tabular}

$\dagger$ Tiempo desde que se detectó la infección por VIH por primera vez hasta que fue reclutado en el estudio

n: número de participantes; DE: desviación estándar.

tativa: edad, tiempo de diagnóstico (años), número de parejas sexuales en el último año, número de parejas sexuales en los últimos seis meses, recuento de células CD4, CD8 y CD3, carga viral (copias/mL), carga viral $(\log 10)$. Los resultados de resistencia, carga viral y CD4/CD8 se reportaron en el sistema NetLab (https://www.netlab.ins.gob.pe/FrmNewLogin.aspx) del INS, para la atención de los participantes a cargo de sus médicos tratantes.

El proyecto fue aprobado por el Comité Institucional de Ética en Investigación del INS con código OI-094-13.

\section{HALLAZGOS}

Se reclutaron 135 participantes con diagnóstico de VIH provenientes de diferentes regiones del país (Tabla 1). La población se caracterizó por ser mayoritariamente masculina $(80,7 \%)$ con una edad media de 35,1 $\pm 10,8$. También se encontró que los pacientes residentes en Ica presentaron el mayor tiempo de diagnóstico por VIH (4,00 \pm 5,66 años) mientras que los de San Martín tuvieron un tiempo de diagnóstico más reciente (0,90 \pm 0,5 años). El estado virológico e inmunológico de los participantes reveló 247 150,8 \pm 39 194,7 copias de ARN/mL y recuento de linfocitos $\mathrm{CD} 4$ de $282,6 \pm 222,7$ células/mL, con un $33,3 \%$ de pacientes en fase sida y 90,7\% de ITS en la población masculina.

Se caracterizó el genotipo de 132 participantes. Se excluyeron tres muestras de VIH debido a que no fueron reactivas a las pruebas de RT-PCR y secuenciamiento, tanto por el sistema in house como por el comercial. La frecuencia de RT de la población estudiada fue de 9,8\% (13/132), con cinco casos en Lima, cuatro en San Martín, dos en Lambayeque y uno en La Libertad y Piura, respectivamente.

Analizando la RT por departamento y de acuerdo con el tipo de ARV, se observó una mayor frecuencia para el grupo de inhibidor no nucleósido de la transcriptasa reversa (INNTR) $(6,1 \%, 8 / 132)$, siendo Lima y San Martín los departamentos que reportaron el mayor índice (Tabla 2). Las mutaciones de resistencia a ARV fueron principalmente asociadas a INNTR ( $\mathrm{n}=10 ; 7,6 \%)$, predominando la mutación E138A (2,3\%) que confiere un bajo nivel de resistencia a rilpivirina (RPV) (Tabla 3). Asimismo, se observó la mutación K103N (1,5\%) asociada a un alto nivel de resistencia a efavirenz y nevirapina. El análisis de los factores socioepidemiológicos reveló que la media del número de parejas sexuales en el último año fue mayor en aquellos pacientes que presentaron RT $(10,8 \pm 27,5)$ (Tabla 4).

\section{DISCUSIÓN}

De acuerdo con nuestros hallazgos la frecuencia de RT en el Perú (9,8\%), corresponde a un nivel moderado de resistencia según la OMS ${ }^{(16)}$, similar al de otros países de LAC (de $8,2 \%$ a $12,8 \%$ ) reportados entre 2014 y $2016^{(4)}$.

A diferencia de nuestro estudio, Lama et al. ${ }^{(9)}$ reportaron una frecuencia de RT de 3,3\%, a pesar de que la población de estudio correspondió a HSH, la cual se caracteriza por presentar comportamiento sexual de alto riesgo de transmisión 
Tabla 2. Frecuencia de genotipo resistente de VIH a los antirretrovirales según departamento y de acuerdo con el grupo de antirretroviral empleado.

\begin{tabular}{|c|c|c|c|c|c|}
\hline Departamento & $\mathbf{n}$ & $\begin{array}{c}\text { Resistencia a algún } \\
\text { ARV } \\
\text { n (\%) }\end{array}$ & $\begin{array}{c}\text { Resistencia a IP } \\
\text { n (\%) }\end{array}$ & $\begin{array}{c}\text { Resistencia a INTR } \\
\text { n (\%) }\end{array}$ & $\begin{array}{c}\text { Resistencia a INN- } \\
\text { TR } \\
\text { n (\%) }\end{array}$ \\
\hline Arequipa & 6 & $0(0,0)$ & $0(0,0)$ & $0(0,0)$ & $0(0,0)$ \\
\hline Ica & 3 & $0(0,0)$ & $0(0,0)$ & $0(0,0)$ & $0(0,0)$ \\
\hline Junín & 10 & $0(0,0)$ & $0(0,0)$ & $0(0,0)$ & $0(0,0)$ \\
\hline La Libertad & 7 & $1(14,3)$ & $0(0,0)$ & $0(0,0)$ & $1(14,3)$ \\
\hline Lambayeque & 15 & $2(13,3)$ & $0(0,0)$ & $0(0,0)$ & $2(13,3)$ \\
\hline Lima/Callao & 40 & $5(12,5)$ & $1(2,5)$ & $0(0,0)$ & $4(10,0)$ \\
\hline Loreto & 17 & $0(0,0)$ & $0(0,0)$ & $0(0,0)$ & $0(0,0)$ \\
\hline Piura & 3 & $1(33,3)$ & $1(33,3)$ & $0(0,0)$ & $0(0,0)$ \\
\hline San Martín & 31 & $4(12,9)$ & $2(6,5)$ & $1(3,2)$ & $1(3,2)$ \\
\hline Total & 132 & $13(9,8)$ & $4(3,0)$ & $1(0,8)$ & $8(6,1)$ \\
\hline
\end{tabular}

n: número de participantes; ARV: antirretroviral; IP: inhibidor de proteasa; INTR: inhibidor nucleósido de la transcriptasa reversa; INNTR: inhibidor no nucleósido de la transcriptasa reversa.

de VIH/ITS ${ }^{(17)}$. Este valor probablemente guarda relación con el período en el que se ejecutó el estudio, donde el TAR en el Perú recién empezaba a implementarse, poco después de que la OMS inició el monitoreo de la emergencia de resistencia a drogas ${ }^{(16)}$.

De otro lado, las mutaciones identificadas en este estudio se relacionaron en su mayoría con la resistencia a los

Tabla 3. Frecuencia de mutaciones de resistencia identificadas según el tipo de antirretroviral.

\begin{tabular}{lccc}
\hline Mutación asociada & $\mathbf{n}(\%)$ & ARV & NR \\
\hline IP & & & \\
Q58E & $3(2,3)$ & FPV & Bajo \\
L10F & $1(0,7)$ & TPV & Bajo \\
Total & $4(3,0)$ & & \\
INTR & & & \\
T215N & $1(0,7)$ & AZT, A4T & Bajo \\
Total & $1(0,7)$ & & \\
INNTR & & & \\
E138A & $3(2,3)$ & RPV & Bajo \\
K103N & $2(1,5)$ & EFV, NVP & Alto \\
V106I & $2(1,5)$ & DOR & Bajo \\
V108I & $2(1,5)$ & NVP & Bajo \\
A98G & $1(0,8)$ & DOR, EFV, RPV & Bajo \\
Total & $10(7,6)$ & & \\
\hline
\end{tabular}

IP: inhibidores de proteasa; INTR: inhibidores nucleósidos de transcriptasa reversa; INNTR: inhibidores no nucleósidos de transcriptasa reversa; n: número total de mutaciones; ARV: antirretroviral afectado por la mutación; NR: nivel de resistencia según clasificación del HIV db Programa de HIV Drug Resistance Data Base de la Universidad de Stanford; FPV: fosamprenavir; TPV: tripnavir; AZT: zidovudina; D4T: estavudina; NVP: nevirapina; RPV: rilpivirina; EFV: efavirenz; DOR: doravirina.
INNTR, en concordancia con la resistencia descrita recientemente en población HSH y transgénero de Lima ${ }^{(12)}$ y con diferentes reportes en países de LAC ${ }^{(5,6)}$. Asimismo, la RT en comparación con INNTR podría estar relacionada con la resistencia adquirida en población peruana, donde recientemente identificamos una alta prevalencia de resistencia al INNTR $^{(18)}$.

De otro lado, los estudios reportados por Soria et al. ${ }^{(10,11)}$ realizados en la ciudad de Lima revelaron considerables diferencias en la RT en dos períodos de tiempo, desde un nivel bajo (1\% entre 2007 y 2009) hasta un nivel elevado (14\% entre 2014 y 2015), lo cual sugiere que la RT estaría experimentando un incremento a través del tiempo, tal como se ha reportado en otros países, donde la RT se elevó de bajo a moderado en solo seis años ${ }^{(16)}$. Estos datos sugieren plantear estudios longitudinales o bien vigilancias periódicas nacionales que permitan identificar los cambios de la RT en el tiempo, con el fin de tomar medidas de prevención apropiadas y reducir la trasmisión de VIH en la población.

Cabe indicar que dentro de los factores socioepidemiológicos estudiados, hemos identificado que la media del número de compañeros sexuales en el último año fue mayor en el grupo que presentó RT. Si bien estos hallazgos son descriptivos, sugieren que la promiscuidad sexual podría estar jugando un rol importante en la transmisión de cepas resistentes de VIH, lo cual podría explicar la alta prevalencia de RT encontrada en población HSH y transgénero de Lima ${ }^{(12)}$ $y$ en otros estudios relacionados ${ }^{(4,5)}$.

La principal limitación del estudio es el tiempo transcurrido desde que se realizó la colección de las muestras a la actualidad ( $>5$ años). Sin embargo, consideramos que los hallazgos presentados en este artículo ayudarán a comprender mejor el fenómeno de la RT en el Perú, lo que permitirá 
Tabla 4. Resistencia transmitida a los antirretrovirales en VIH-1 según características socioepidemiológicas, virológicas e inmunológicas.

\begin{tabular}{|c|c|c|c|}
\hline \multirow{2}{*}{ Características } & \multicolumn{2}{|c|}{ Sin resistencia } & \multirow{2}{*}{$\begin{array}{c}\text { Con resistencia }(n=13) \\
\text { Media } \pm \text { DE }\end{array}$} \\
\hline & n & Media \pm DE & \\
\hline Edad & 116 & $35,4 \pm 10,9$ & $32,9 \pm 9,5$ \\
\hline Tiempo de diagnóstico (años) & 114 & $1,7 \pm 2,0$ & $1,3 \pm 0,9$ \\
\hline Número de parejas sexuales en el último año & 111 & $6,7 \pm 29,9$ & $10,8 \pm 27,5$ \\
\hline Número de parejas sexuales en los últimos 6 meses & 111 & $3,7 \pm 17,6$ & $0,8 \pm 1,3$ \\
\hline Recuento de células CD4 (células/ $\mu \mathrm{L}$ ) & 117 & $274,0 \pm 220,3$ & $274,7 \pm 170,4$ \\
\hline Recuento de células CD8 (células/ $\mu \mathrm{L}$ ) & 117 & $1054,9 \pm 561,9$ & $804,9 \pm 573,8$ \\
\hline Recuento de células CD3 (células/ $\mu \mathrm{L}$ ) & 117 & $1380,4 \pm 693,2$ & $1141,5 \pm 718,2$ \\
\hline Carga viral (copias/mL) & 118 & $259740,2 \pm 407699,8$ & $151753,7 \pm 199932,0$ \\
\hline Carga viral $(\log 10)$ & 118 & $5,0 \pm 0,7$ & $4,8 \pm 0,7$ \\
\hline
\end{tabular}

n: número de participantes; DE: desviación estándar.

plantear nuevas políticas de salud pública enfocadas a generar cambios en las estrategias de TAR de primera línea a través de la identificación temprana del genotipo resistente.

En conclusión, el presente estudio muestra que la RT en el Perú es moderada, principalmente a INNTR, altamente frecuente en departamentos de la costa y selva, y en personas con varias parejas sexuales. En consecuencia, se requieren desarrollar nuevos estudios de vigilancia que permitan conocer la situación actual de la RT en el Perú, además de sus factores asociados.

Agradecimientos: Los autores agradecen la colaboración de Reyna Mayta, Lita Alarcón (Hospital Regional Las Mercedes, Lambayeque) Margarita Pinedo (Hospital de Ferreñafe, Lambayeque), Nidia Calderón (Hospital Regional de San Martín), Heriberto Arévalo y Yolanda Monteza (Laboratorio Regional de San Martín), Raul Montalvo y Omar Orellana (Hospital Regional de Junín), Juan Díaz, Nancy Brizuela (Hospital Regional de Ica), Angélica García, Mariza Rojas, (Hospital Docente de Trujillo), María Gonzales, Lucía Gonzales y Gonzalo Castañeda (Hospital Regional de Arequipa), Cesar Ramal (Hospital Regional de Iquitos), Cesar Guerrero (Hospital Regional de Piura), María Dedios

\section{REFERENCIAS BIBLIOGRÁFICAS}

1. United Nations Programme on HIV/AIDS. UNAIDS Data 2018. [Internet]. Geneva: UNAIDS; 2018 [citado el 17 de agosto de 2020]. Disponible en: http://www.unaids.org/sites/default/files/media_asset/ unaids-data-2018_en.pdf.

2. Clutter DS, Jordan MR, Bertagnolio S, Shafer RW. HIV-1 drug resistance and resistance testing. Infect Genet Evol. 2016;46:292-307. doi: 10.1016/j. meegid.2016.08.031.

3. Rhee SY, Jordan MR, Raizes E, Chua A, Parkin N, Kantor R, et al. HIV-1 Drug Resistance Mutations: Potential Applications for Point-of-Care Genotypic Resistance Testing. PLoS One. 2015;10(12):e0145772. doi: 10.1371/journal.pone.0145772.

4. Avila-Rios S, Sued O, Rhee SY, Shafer RW, Reyes-Teran G, Ravasi G. Surveillance of HIV Transmitted Drug Resistance in Latin America and
(Hospital Santa Rosa), Eduardo Sánchez (Hospital Hipólito Unanue), Jorge Arévalo (Hospital Dos de Mayo), Patricia Gonzales (Hospital María Auxiliadora), Augusto Escalante (Hospital San José), Miguel Estela (Hospital de Huacho) por su esfuerzo y apoyo en el proceso de reclutamiento, encuesta y toma de muestra de los participantes del estudio, sin ellos la presente investigación no hubiera podido realizarse. Asimismo, los autores agradecen el asesoramiento del Dr. Gilmer Solís Sánchez en los análisis e interpretación de los datos estadísticos.

Contribuciones de autoría: CAY participó en la concepción del artículo, análisis e interpretación de datos, redacción y aprobación de la versión final del artículo. GFV, SE, MGY, GS, LGF, RDVB, CB, DSA, EM, MA, SR y FC participaron en el análisis e interpretación de datos, revisión crítica y aprobación de la versión final del artículo. SE, MGY, DSA, EM, MA, SR y FC participaron en la recolección de datos. Todos los autores asumen responsabilidad de los contenidos del manuscrito.

Financiamiento: El artículo fue financiado íntegramente por el Instituto Nacional de Salud.

Conflictos de interés: Los autores declaran no tener conflictos de interés.

the Caribbean: A Systematic Review and Meta-Analysis. PLoS One. 2016;11(6):e0158560. doi: 10.1371/journal.pone.0158560.

5. Gupta RK, Gregson J, Parkin N, Haile-Selassie H, Tanuri A, Andrade Forero L, et al. HIV-1 drug resistance before initiation or re-initiation of first-line antiretroviral therapy in low-income and middle-income countries: a systematic review and meta-regression analysis. Lancet Infect Dis. 2018;18(3):346-355. doi: 10.1016/S1473-3099(17)30702-8.

6. Wang X, He C, Xing H, Liao L, Xu X, He J, et al. Short communication: emerging transmitted HIV type 1 drug resistance mutations among patients prior to start of first-line antiretroviral therapy in middle and low prevalence sites in China. AIDS Res Hum Retroviruses. 2012;28(12):16379. doi: 10.1089/aid.2012.0164.

7. Chung MH, Beck IA, Dross S, Tapia K, Kiarie JN, Richardson BA, et al. Oligonucleotide ligation assay detects HIV drug resistance as- 
sociated with virologic failure among antiretroviral-naive adults in Kenya. J Acquir Immune Defic Syndr. 2014;67(3):246-53. doi: 10.1097/ QAI.0000000000000312.

8. Hong SY, Jonas A, DeKlerk M, Shiningavamwe A, Desta T, Badi A, et al. Population-based surveillance of HIV drug resistance emerging on treatment and associated factors at sentinel antiretroviral therapy sites in Namibia. J Acquir Immune Defic Syndr. 2015;68(4):463-71. doi: 10.1097/ QAI.0000000000000509.

9. Lama JR, Sanchez J, Suarez L, Caballero P, Laguna A, Sanchez JL, et al. Linking HIV and antiretroviral drug resistance surveillance in Peru: a model for a third-generation HIV sentinel surveillance. J Acquir Immune Defic Syndr. 2006;42(4):501-5. doi: 10.1097/01.qai.0000221677.29693.dd.

10. Soria J, Bull M, Mitchell C, La Rosa A, Dross S, Kraft K, et al. Transmitted HIV resistance to first-line antiretroviral therapy in Lima, Peru. AIDS Res Hum Retroviruses. 2012;28(4):333-8. doi: 10.1089/aid.2011.0131.

11. Soria J, Mugruza R, Levine M, León SR, Arévalo J, Ticona E, et al. Pretreatment HIV Drug Resistance and Virologic Outcomes to First-Line Antiretroviral Therapy in Peru. AIDS Res Hum Retroviruses. 2019;35(2):150-154. doi: 10.1089/AID.2018.0239.

12. Trebelcock WL, Lama JR, Duerr A, Sanchez H, Cabello R, Gilada T, et al. HIV pretreatment drug resistance among cisgender MSM and transgender women from Lima, Peru. J Int AIDS Soc. 2019; 22(11):e25411. doi: 10.1002/jia2.25411.
13. Ministerio de Salud. Sala situacional VIH/SIDA, CDC Perú [Internet]. Lima: Dirección General de Epidemiología, MINSA; 2020 [citado el 17 de agosto de 2020]. Disponible en: https://www.dge.gob.pe/vih/.

14. Chaturbhuj DN, Nirmalkar AP, Paranjape RS, Tripathy SP. Evaluation of a cost effective in-house method for HIV-1 drug resistance genotyping using plasma samples. PLoS One. 2014;9(2):e87441. doi: 10.1371/journal. pone. 0087441 .

15. Woods CK, Brumme CJ, Liu TF, Chui CK, Chu AL, Wynhoven B, et al. Automating HIV drug resistance genotyping with RECall, a freely accessible sequence analysis tool. J Clin Microbiol. 2012; 50(6):1936-42. doi: 10.1128/JCM.06689-11.

16. World Organization Health. The HIV drug resistance report - 2012 [Internet]. Geneva: WHO; 2012 [citado el 17 de agosto de 2020]. Disponible en: https://www.who.int/hiv/pub/drugresistance/report2012/en/.

17. Cárcamo CP, Campos PE, García PJ, Hughes JP, Garnett GP, Holmes KK, et al. Prevalences of sexually transmitted infections in young adults and female sex workers in Peru: a national population-based survey. Lancet Infect Dis. 2012; 12(10), 765-773. doi: 10.1016/S1473-3099(12)70144-5.

18. Yabar CA, Vilcarino G, Yaya M, Espetia S, Acuña M, Mamani E, et al. Multiple Resistance and Unusual Mutations from HIV-1 Infecting Peruvian Patients with Highly Active Antiretroviral Therapy. HIV AIDS Res J. 2018;1:2. 